\title{
An Interval Graph with Alternate Cliiques of Size 3- Signed Roman Domination
}

\author{
M. Reddappa \\ Research scholar \\ Dept. of Mathematics \\ S.V.University, Tirupati-517502
}

\author{
C. Jaya Subba Reddy \\ Asst. Professor \\ Dept. of Mathematics \\ S.V.University, Tirupati-517502
}

\author{
B. Maheswari \\ Professor(Rtd.) \\ Dept. of Applied Mathematics \\ S.P.M.V.Visvavidyalayam \\ Tirupati-517502
}

\begin{abstract}
Today graph theory is one of the most flourishing braches of modern mathematics. Graphs are useful in enhancing the understanding of the organization and behavioural characteristics of complex system. The study of domination in graphs originated around 1850 has become the source of interest to the researchers.

Interval graphs have drawn the attention of many researchers for over 40 years. They form a special class of graphs with many interesting properties and revealed their practical relevance for modeling problems arising in the real world. The theory of domination in graphs introduced by Ore [11] and Berge [6] is fast growing area of research in graph theory today. An introduction and an extensive overview on domination in graphs and related topics is surveyed and detailed in the two books by Haynes et.al. [1, 2].
\end{abstract}

The concept of signed Roman dominating function was introduced by Ahangar et al. [4]. They present various lower and upper bounds on the signed Roman domination number of a graph and characterized the graphs which have these bounds. The minimal signed Roman dominating functions of corona product graph of a path with a star is studied by Siva Parvathi [13].

In this paper a study of signed Roman domination in an interval graph with alternate cliques of size 3 is carried out.

\section{Keywords}

Signed Roman dominating function, Signed Roman domination number, Interval family, Interval graph.

\section{INTRODUCTION}

Domination in graphs has been studied extensively in recent years and it is an important branch of Graph Theory. Allan, R.B. and Laskar, R.C.[5], Cockayne, E.J.andHedetniemi, S.T [7] and many others have studied various domination parameters of graphs.

Let $G(V, E)$ be a graph. A subset $D$ of $V$ is said to be a dominating set of $G$ if every vertex in $V-D$ is adjacent to a vertex in $D$. The minimum cardinality of a dominating set is called as the domination number and is denoted by $\gamma(G)$.

We consider finite graphs without loops and multiple edges.

\section{SIGNED ROMAN DOMINATING FUNCTION}

The concept of Signed dominating function was introduced by Dunbar et al. [3]. There is a variety of possible applications for this variation of domination. By assigning the values -1 or +1 to the vertices of a graph we can model such things as networks of positive and negative electrical charges, networks of positive and negative spins of electrons and networks of people or organizations in which global decisions can be made.

The Roman dominating function of a graph $G$ was defined by Cockayne et.al [8]. The definition of a Roman dominating function was motivated by an article in Scientific American by Ian Stewart [9] entitled "Defend The Roman Empire!" and suggested by even earlier byReVelle [12]. Domination number and Roman domination number in an interval graph with consecutive cliques of size 3 are studied by Jaya Subba Reddy. C, Reddappa. M andMaheswari. B [10].

A Roman dominating function on a graph $G(V, E)$ is a function $f: V \rightarrow\{0,1,2\}$ satisfying the condition that every vertex $u$ for which $f(u)=0$ is adjacent to at least one vertex $v$ for which $f(v)=2$.The weight of a Roman dominating function is the value $f(V)=\sum_{v \in V} f(v)$. The minimum weight of a Roman dominating function on a graph $G$ is called as the Roman domination number of $G$. It is denoted by $\gamma_{R}(G)$. If $\gamma_{R}(G)=2 \gamma(G)$ then $G$ is called a Roman graph.

Let $G=(V, E)$ be a graph. A signed Roman dominating function on the graph $G$ is a function $f: V \rightarrow$ $\{-1,1,2\}$, which satisfies the following two conditions:

(i) For each $u \in V, \sum_{v \in N[u]} f(v) \geq 1$;

(ii) Each vertex $u$ for which $f(u)=-1$ is adjacent to at least one vertex $v$ for which $f(v)=2$.

Thevalue $f(V)=\sum_{u \in v} f(u) \quad$ is called the weight of the function $f$, and it is denoted by $w(f)$. The signed Roman domination number of $G, \gamma_{s R}(G)$ is the minimum weight of a signed Roman domination number on $G$.

Each signed Roman dominating function $f$ on $G$ is uniquely determined by the ordered partition $\left(V_{-1}, V_{1}, V_{2}\right)$ of $V(G)$, where $\quad V_{i}=\{v \in V / f(v)=i\}$ for $i=-1,1,2$. Then $w(f)=-\left|V_{-1}\right|+\left|V_{1}\right|+2\left|V_{2}\right|$.

There exists a 1-1 correspondence between the functions $f: V \rightarrow\{-1,1,2\}$ and the ordered partition $\left(V_{-1}, V_{1}, V_{2}\right)$ of $V$. Thus we write $f=\left(V_{-1}, V_{1}, V_{2}\right)$.

\section{INTERVAL GRAPH}

Let $I=\left\{I_{1}, I_{2}, I_{3}, \ldots \ldots \ldots . I_{n}\right\}$ be an interval family, where each $I_{i}$ is an interval on the real line and $I_{i}=\left[a_{i}, b_{i}\right]$ for $i=$ $1,2,3, \ldots \ldots . n$. Here $a_{i}$ is called the left end point and $b_{i}$ is called the right end point of $I_{i}$. Without loss of generality, we 
assume that all end points of the intervals in $I$ are distinct numbers between 1 and $2 \mathrm{n}$. Two intervals $i=\left[a_{i}, b_{i}\right]$ and $j=$ $\left[a_{j}, b_{j}\right]$ are said to intersect each other if either $a_{j}<b_{i}$ or $a_{i}<b_{j}$. The intervals are labelled in the increasing order of their right end points.

Let $G(V, E)$ be a graph. $G$ is called an interval graph if there is a 1-1 correspondence between $V$ and $I$ such that two vertices of $G$ are joined by an edge in $E$ if and only if their corresponding intervals in $I$ intersect. If $i$ is an interval in $I$ the corresponding vertex in $G$ is denoted by $v_{i}$.

Consider the following interval family.

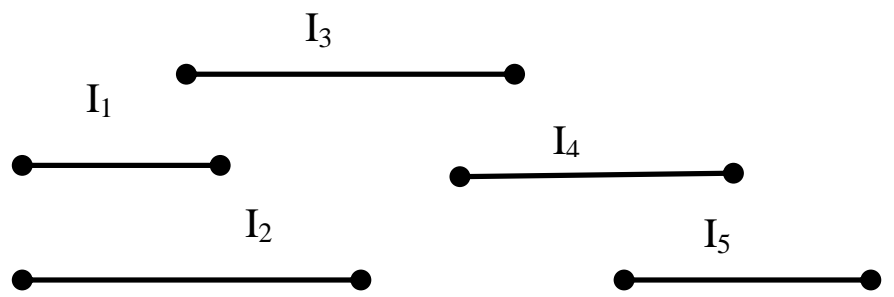

The corresponding interval graph is given by

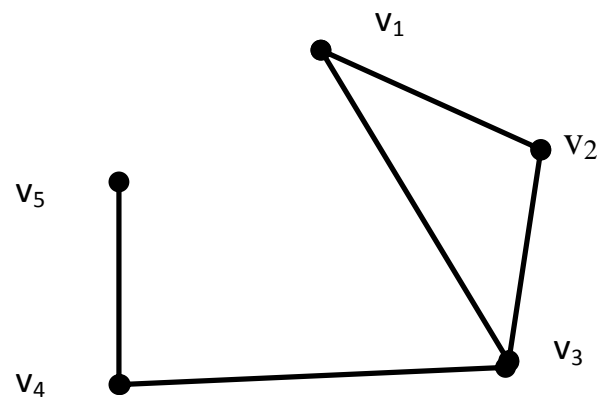

Consider the following interval family.

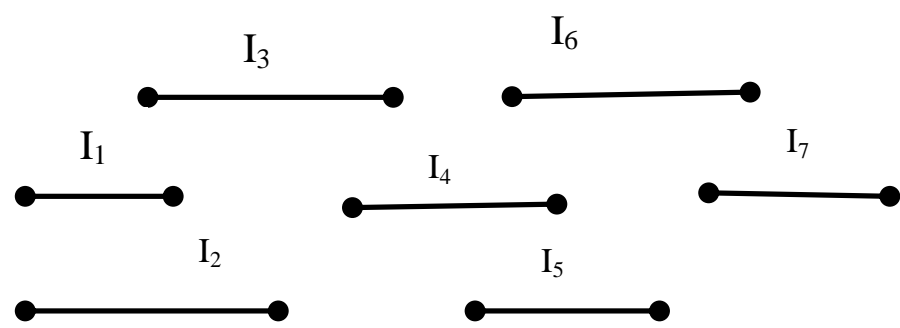

The corresponding interval graph is given by

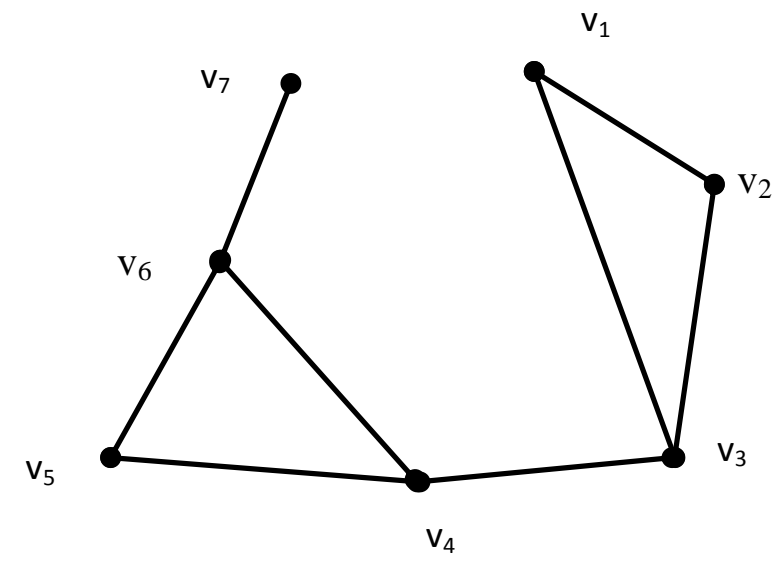

Consider the following interval family.

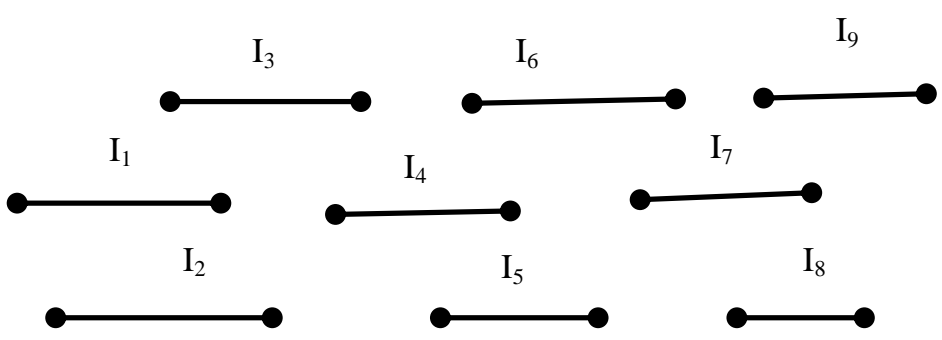

The corresponding interval graph is given by

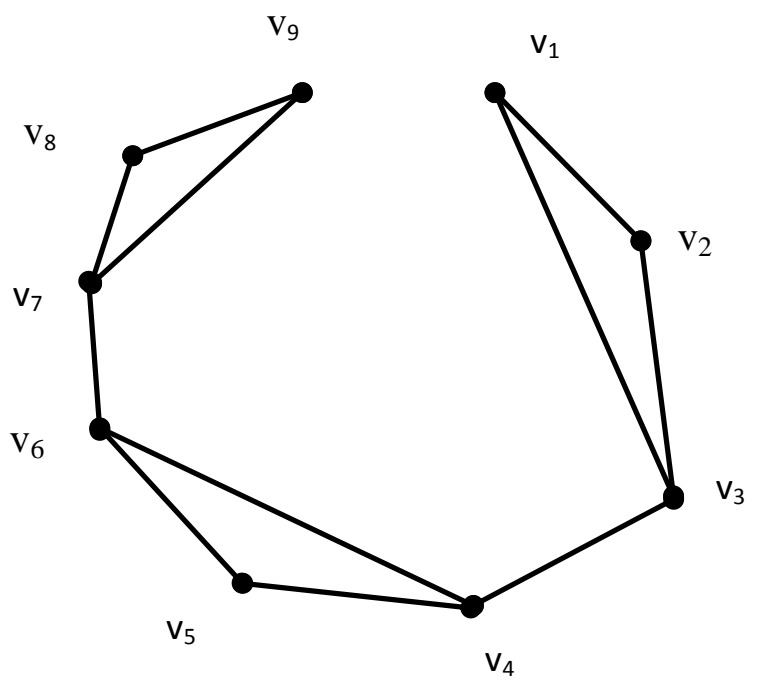

In what follows we consider interval graphs of this type. We observe that when $n=3 k+3$ then the interval graph has adjacent cliques of size $3, k=1,2,3 \ldots \ldots$ and when $n=3 k+$ 2 then the interval graph has adjacent cliques of size 3 and the last clique has two adjacent edges and when $n=3 k+4$ then the interval graph has adjacent cliques of size 3 and the last clique is adjacent with one edge, $k=1,2,3 \ldots \ldots$. We denote this type of interval graph by $\mathcal{G}$. The signed Roman domination is studied in the following for the interval graph $\boldsymbol{G}$.

\section{RESULTS}

Theorem 4.1: Let $\boldsymbol{G}$ the Interval graph with $n$ vertices, where $n \geq 5$. Then the signed Roman domination of $\mathcal{G}$ is

$\gamma_{s R}(\boldsymbol{G})=2 k+1$ for $=3 k+2,3 k+4$,

$=2 k+2$ for $n=3 k+3$ 
where $k=1,2,3 \ldots \ldots$..respectively.

Proof: Let $\mathcal{G}$ be the interval graph with $n$ vertices, where $n \geq 5$.

Let the vertex set of $\boldsymbol{g}$ be $\left\{v_{1}, v_{2}, v_{3}, v_{4} \ldots \ldots \ldots \ldots \ldots v_{n}\right\}$.

Case 1: Suppose $n=3 k+2$, where $k=1,2,3 \ldots \ldots \ldots$.

Let $f: V \rightarrow\{-1,1,2\}$ and let $\left(V_{-1}, V_{1}, V_{2}\right)$ be the ordered partition of $V$ induced by $f$ where $V_{i}=\{v \in V / f(v)=i\}$ for $i=-1,1,2$. Then there exist a 1-1 correspondence between the functions $f: V \rightarrow\{-1,1,2\}$ and the ordered partition $\left(V_{-1}, V_{1}, V_{2}\right)$ of $V$.Thus we write $f=$ $\left(V_{-1}, V_{1}, V_{2}\right)$.

Let $V_{1}=\left\{v_{1}, v_{4}, \ldots \ldots \ldots \ldots \ldots, v_{n-10}, v_{n-7}, v_{n-4}\right\}$;

$V_{2}=\left\{v_{3}, v_{6}, \ldots \ldots \ldots \ldots \ldots, v_{n-5}, v_{n-2}, v_{n}\right\}$;

$V_{-1}=\left\{v_{2}, v_{5}, \ldots \ldots \ldots \ldots \ldots, v_{n-6}, v_{n-3}, v_{n-1}\right\}$.

It was shown in [10] that $V_{2}$ is a minimum dominating set of $\boldsymbol{G}$. Further the set $V_{2}$ dominates $V_{-1}$. That is, every vertex $u$ such that $f(u)=-1$ is adjacent to some vertex $v$ with $f(v)=2$.

Therefore $f=\left(V_{-1}, V_{1}, V_{2}\right)$ becomes a signed Roman dominating function of $\boldsymbol{G}$.

Now $\left|V_{1}\right|=k,\left|V_{2}\right|=k+1,\left|V_{-1}\right|=k+1$.

Therefore

$\sum_{v \in V} f(v)=\sum_{v \in V_{-1}} f(v)+\sum_{v \in V_{1}} f(v)+\sum_{v \in V_{2}} f(v)$.

$=-k-1+k+2 k+2=2 k+1$.

Let $g=\left(V_{-1}^{\prime}, V_{1}^{\prime}, V_{2}^{\prime}\right)$ be a signed Roman dominating function of $\mathcal{G}$, where $V_{2}^{\prime}$ dominates $V_{-1}^{\prime}$. Then $g(V)=$ $\sum_{v \in V^{\prime}} g(v)=\sum_{v \in V_{-1}^{\prime}} g(v)+\sum_{v \in V_{1}^{\prime}} g(v)+\sum_{v \in V_{2}^{\prime}} g(v)$

$$
=-\left|V_{-1}^{\prime}\right|+\left|V_{1}^{\prime}\right|+2\left|V_{2}^{\prime}\right|
$$

Since $V_{2}$ is a minimum dominating set of $\mathcal{G}$, we have $\left|V_{2}\right| \leq$ $\left|V_{2}^{\prime}\right|$. This implies that $g(V)=-\left|V_{-1}^{\prime}\right|+\left|V_{1}^{\prime}\right|+2\left|V_{2}^{\prime}\right| \geq$ $-\left|V_{-1}\right|+\left|V_{1}\right|+2\left|V_{2}\right|=f(V)$.

Therefore $f(V)$ is a minimum weight of $\boldsymbol{g}$, Where $f\left(V_{-1}, V_{1}, V_{2}\right)$ is a signed Roman dominating function.

Thus $\gamma_{s R}(\mathcal{G})=2 k+1$.

Case 2: Suppose $n=3 k+3$, where $k=1,2,3 \ldots \ldots \ldots$.

Now we proceed as in Case 1.

Let $V_{1}=\left\{v_{1}, v_{4}, \ldots \ldots \ldots \ldots \ldots, v_{n-8}, v_{n-5}, v_{n-2}\right\}$;

$V_{2}=\left\{v_{3}, v_{6}, \ldots \ldots \ldots \ldots \ldots, v_{n-6,}, v_{n-3}, v_{n}\right\}$;

$V_{-1}=\left\{v_{2}, v_{5}, \ldots \ldots \ldots \ldots \ldots, v_{n-7}, v_{n-4}, v_{n-1}\right\}$.

Clearly $V_{2}$ is a minimum dominating set of $\boldsymbol{G}$. Here we observe that the set $V_{2}$ dominates $V_{-1}$. Therefore $f=$ $\left(V_{-1}, V_{1}, V_{2}\right)$ becomes a signed Roman dominating function of $\mathcal{G}$.

Now $\left|V_{1}\right|=k+1,\left|V_{2}\right|=k+1,\left|V_{-1}\right|=k+1$.
Therefore

$\sum_{v \in V} f(v)=\sum_{v \in V_{-1}} f(v)+\sum_{v \in V_{1}} f(v)+\sum_{v \in V_{2}} f(v)$.

$=-k-1+k+1+2 k+2=2 k+2$.

If $g=\left(V_{0}^{\prime}, V_{1}^{\prime}, V_{2}^{\prime}\right)$ is a Roman dominating function of $\boldsymbol{G}$, then it follows as in Case 1, that $f(V)$ is a minimum weight of $\mathcal{G}$ for the signed Roman dominating function $f\left(V_{0}, V_{1}, V_{2}\right)$.

Thus $\gamma_{S R}(G)=2 k+2$.

Case 3: Suppose $n=3 k+4$, where $k=1,2,3 \ldots \ldots \ldots$.

Now proceed as in Case 1.

Let $V_{1}=\left\{v_{1}, v_{4}, \ldots \ldots \ldots \ldots \ldots, v_{n-9}, v_{n-6,}, v_{n-3}\right\}$;

$V_{2}=\left\{v_{3}, v_{6}, \ldots \ldots \ldots \ldots \ldots, v_{n-7}, v_{n-4}, v_{n-1}\right\}$;

$V_{-1}=\left\{v_{2}, v_{5}, \ldots \ldots \ldots \ldots \ldots, v_{n-5}, v_{n-2}, v_{n}\right\}$.

Obviously $V_{2}$ is a minimum dominating set of $\mathcal{G}$. Further the set $V_{2}$ dominates $V_{-1}$.

Therefore $f=\left(V_{-1}, V_{1}, V_{2}\right)$ becomes a signed Roman dominating function of $\boldsymbol{G}$.

Now $\left|V_{1}\right|=k+1,\left|V_{2}\right|=k+1,\left|V_{-1}\right|=k+2$.

Therefore

$\sum_{v \in V} f(v)=\sum_{v \in V_{-1}} f(v)+\sum_{v \in V_{1}} f(v)+\sum_{v \in V_{2}} f(v)$.

$=-k-2+k+1+2 k+2=2 k+1$.

If $g=\left(V_{0}^{\prime}, V_{1}^{\prime}, V_{2}^{\prime}\right)$ is a Roman dominating function of $\mathcal{G}$, then it follows as in Case 1, that $f(V)$ is a minimum weight of $\mathcal{G}$ for the signed Roman dominating function $f\left(V_{0}, V_{1}, V_{2}\right)$.

Thus $\gamma_{s R}(\boldsymbol{G})=2 k+1$.

Theorem 4.2: Let $\mathcal{G}$ be the interval graph with $n$ vertices, where $2<n<6$. Then

$\gamma_{S R}(\boldsymbol{G})=1$ for $n=4$

$=2$ for $n=3$

Proof: Let $\mathcal{G}$ be the interval graph with $n$ vertices, where $2<n<6$

Case 1: Suppose $n=3$. Let $v_{1}, v_{2}, v_{3}$ be the vertices of $\boldsymbol{G}$.

$$
\text { Let } V_{1}=\left\{v_{3}\right\} ; \quad V_{2}=\left\{v_{2}\right\} ; V_{-1}=\left\{v_{1}\right\}
$$

Clearly $V_{2}$ is a minimum dominating set of $\boldsymbol{G}$ and the set $V_{2}$ dominates $V_{-1}$.

Therefore $f=\left(V_{-1}, V_{1}, V_{2}\right)$ is a signed Roman dominating function of $\boldsymbol{G}$.

Therefore

$$
\begin{gathered}
\sum_{v \in V} f(v)=\sum_{v \in V_{-1}} f(v)+\sum_{v \in V_{1}} f(v)+\sum_{v \in V_{2}} f(v) . \\
=-1+1+2 \times 1=2 .
\end{gathered}
$$

Thus $\gamma_{S R}(\boldsymbol{G})=2$.

Case 2: Suppose $n=4$. Let $v_{1}, v_{2}, v_{3}, v_{4}$ be the vertices of $\boldsymbol{G}$. Let $V_{1}=\left\{v_{1}\right\} ; V_{2}=\left\{v_{3}\right\} ; V_{-1}=\left\{v_{2}, v_{4}\right\}$.

Obviously $V_{2}$ is a minimum dominating set of $\mathcal{G}$ and the set $V_{2}$ dominates $V_{-1}$. 
Therefore $f=\left(V_{-1}, V_{1}, V_{2}\right)$ is a signed Roman dominating function of $\boldsymbol{G}$.

\section{Therefore}

$\sum_{v \in V} f(v)=\sum_{v \in V_{-1}} f(v)+\sum_{v \in V_{1}} f(v)+\sum_{v \in V_{2}} f(v)$.

$=-2+1+2 \times 1=1$.

Thus $\gamma_{s R}(\boldsymbol{G})=1$.

Theorem 4.3: Let $\mathcal{G}$ be the interval graph with $n$ vertices, where $n \geq 5$. Then $\gamma_{s R}(\boldsymbol{G})=\gamma(\boldsymbol{G})+k$, for $=3 k+2,3 k+4$ , where $k=1,2,3, \ldots \ldots \ldots$...respectively.

Proof :Let $\mathcal{G}$ be the interval graph with $n$ vertices, where $n \geq 5$.

Then by [10], we have

$\gamma(\mathcal{G})=k+1$ for $n=3 k+2,3 k+4$, where $k=1,2,3, \ldots \ldots$

By Theorem 4.1, we have

$\gamma_{s R}(\boldsymbol{G})=2 k+1, \quad$ for $\quad n=3 k+2,3 k+4, \quad$ where $k=$ $1,2,3 \ldots \ldots \ldots \ldots$

For $n=5 k+2,5 k+4$, where $k=1,2,3 \ldots \ldots \ldots \ldots$

$$
\begin{gathered}
\gamma_{s R}(G)=2 k+1 \\
=(k+1)+k=\gamma(\boldsymbol{G})+k
\end{gathered}
$$

Theorem 4.4: Let $\boldsymbol{G}$ be the interval graph with $n$ vertices, where $n \geq 7$. Then $\gamma_{R}(\boldsymbol{G})=\gamma_{s R}(\boldsymbol{G})+1$, for $=3 k+4$, where $k=1,2,3, \ldots \ldots \ldots$.. respectively.

Proof :Let $\mathcal{G}$ be the interval graph with $n$ vertices, where $n \geq 7$.

Then by [10], we have

$\gamma_{R}(\boldsymbol{G})=2 k+2$, for $n=3 k+4$, where $k=1,2,3 \ldots \ldots \ldots$

Now by Theorem 4.1, we have

$\gamma_{s R}(\boldsymbol{G})=2 k+1$, for $n=3 k+4$, where $k=1,2,3 \ldots \ldots \ldots$

For $n=3 k+4$, where $k=1,2,3 \ldots \ldots \ldots \ldots$

$$
\begin{gathered}
\gamma_{R}(\boldsymbol{G})=2 k+2 \\
=(2 k+1)+1=\gamma_{S R}(\boldsymbol{G})+1
\end{gathered}
$$

Theorem 4.5: Let $\mathcal{G}$ be the interval graph with $n$ vertices, where $n \geq 5$.Then $\gamma_{s R}(\boldsymbol{G})=\gamma_{R}(\boldsymbol{G})$, for $n=3 k+2$, where $k=1,2,3, \ldots \ldots \ldots$...espectively.

Proof :Let $\mathcal{G}$ be the interval graph with $n$ vertices, where $n \geq 5$.

Suppose $n=3 k+2$, where $k=1,2,3, \ldots \ldots \ldots$. respectively.

Then $\gamma_{s R}(\boldsymbol{G})=2 k+1$ and $\gamma_{R}(\boldsymbol{G})=2 k+1$.

Hence $\gamma_{S R}(\boldsymbol{G})=\gamma_{R}(\boldsymbol{G})$.

Theorem 4.6: Let $\mathcal{G}$ be the interval graph with $n$ vertices, where $n \geq 6$. Then $\gamma_{s R}(\boldsymbol{G})=2 \gamma(\boldsymbol{G})$ for $n=3 k+3$, where $k=1,2,3 \ldots \ldots .$. .respectively.

Proof: Let $\boldsymbol{G}$ be the interval graph with $n$ vertices, where $n \geq 6$.

Suppose $n=3 k+3$, and $k=1,2,3 \ldots \ldots$...espectively.

Then by Theorem 4.1, the signed Roman domination number is

$$
\gamma_{S R}(\boldsymbol{G})=2 k+2
$$

$=2(k+1)=2 \gamma(\boldsymbol{G})$

Thus $\gamma_{s R}(\boldsymbol{G})=2 \gamma(\boldsymbol{G})$.

\section{ILLUSTRATIONS}

Illustration 1: $n=7$.
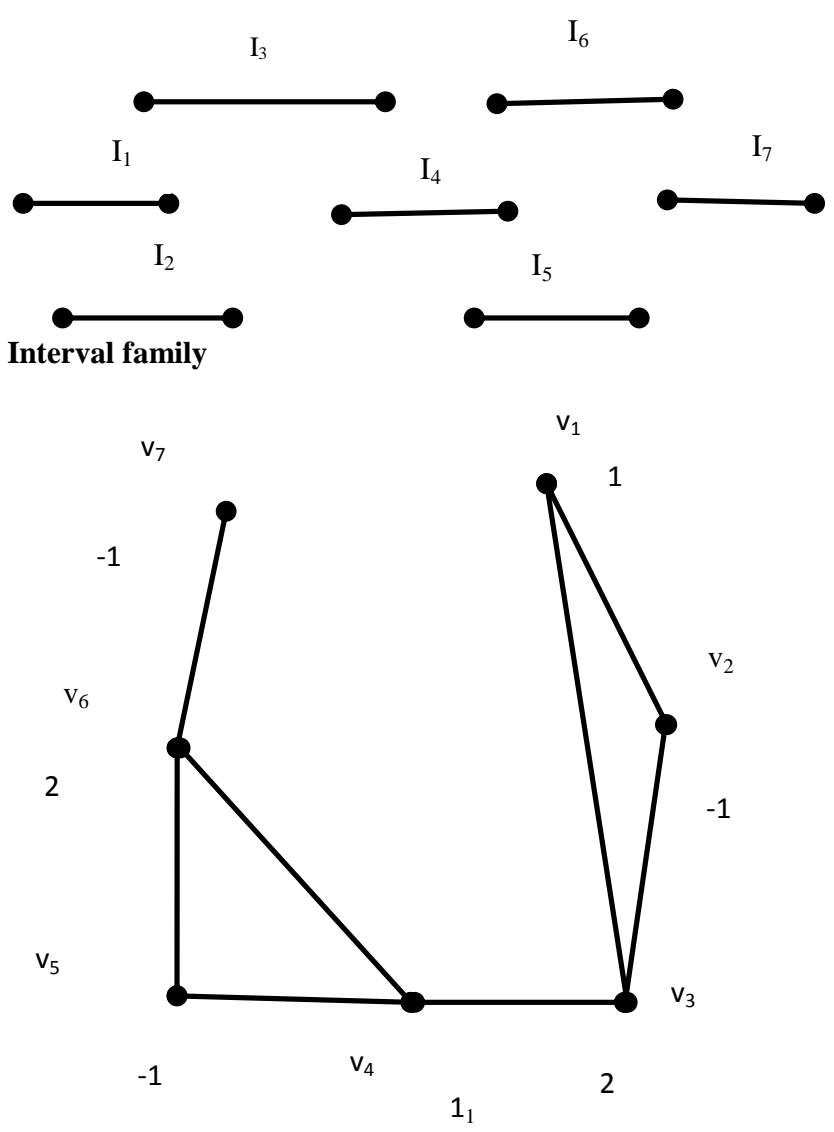

Interval graph $\mathcal{G}$

$$
\begin{aligned}
& \qquad D=\left\{v_{3}, v_{6}\right\} \text { and } \gamma(\boldsymbol{G})=2 . \\
& V_{1}=\left\{v_{1}, v_{4}\right\} ; \quad V_{2}=\left\{v_{3}, v_{6}\right\} ; V_{-1}=\mathrm{V}-\left\{V_{2}\right\}=\left\{v_{2}, v_{5}, v_{7}\right\} \\
& \sum_{v \in V} f(v)=\left|V_{-1}\right| .-1+\left|V_{1}\right| .1+\left|V_{2}\right| .2=-1(3)+ \\
& 1(2)+2(2)=3=f(V) \\
& \text { Therefore } \gamma_{s R}(\boldsymbol{G})=3 .
\end{aligned}
$$

Illustration 2: $n=9$

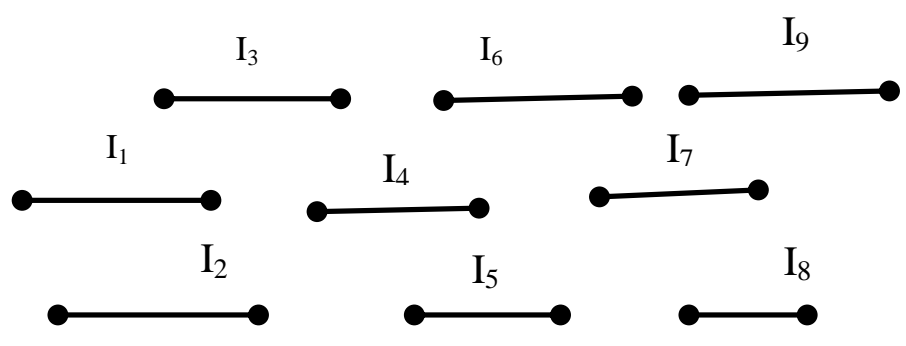

Interval family 


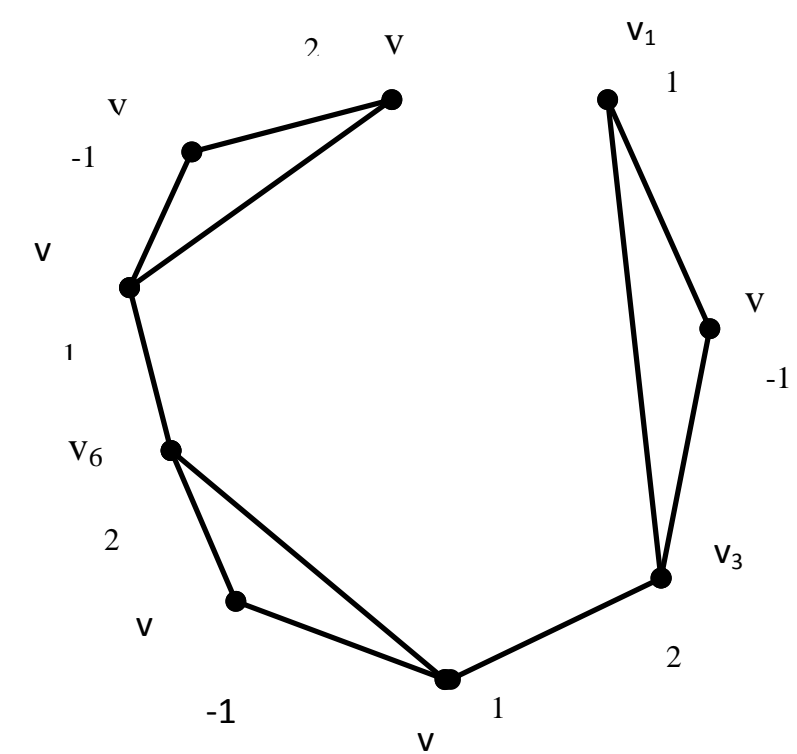

Interval graph $\mathcal{G}$

$D=\left\{v_{3}, v_{6}, v_{9}\right\}$ and $\gamma(\boldsymbol{G})=3$.

$V_{1}=\left\{v_{1}, v_{4}, v_{7}\right\} ; V_{2}=\left\{v_{3}, v_{6}, v_{9}\right\} ; V_{-1}=\left\{v_{2}, v_{5}, v_{8}\right\}$.

$\sum_{v \in V} f(v)=\left|V_{-1}\right| .-1+\left|V_{1}\right| .1+\left|V_{2}\right| .2=-1(3)+$

$1(3)+2(3)=6=f(V)$

Therefore $\gamma_{s R}(\boldsymbol{G})=6$.

\section{REFERENCES}

[1] Haynes, T.W., Hedetniemi, S.T., and Slater, P.J. 1998 Domination in graphs: Advanced Topics, Marcel Dekkar, Inc., New York.

[2] Haynes, T.W., Hedetniemi, S.T., and Slater, P.J.1998 Fundamentals of domination in graphs, Marcel Dekkar, Inc., New York.
[3] Dunbar.J., Hedetniemi.S.T., Henning, M.A. and Slater, P.J. 1995.Signed domination in graphs, in: Y. Alari and A. Schwenk (Eds.), Proc. $7^{\text {th }}$ Internat. Conf.on the Theory and Applications of Graphs, Wiley, New York, $311-321$.

[4] Ahangar, H.A., Henning, M.A., Zhao, Y., Lowenstein, C., Samodivkin, V. 2012. Signed Roman domination in graphs, J.Comb.Optim.

[5] Allan, R.B. and Laskar, R.C. 1978. On domination, Independent domination numbers of a graph Discrete Math., 23, 73-76.

[6] Berge, C. 1980. Graphs and Hyperactive graphs, North Holland, Amsterdam in graphs, Networks, 10,211 - 215.

[7] Cockayne, E.J. and Hedetniemi, S.T. 1977. Towards a theory of domination in graphs. Networks 7, 247 -261.

[8] Cockayne, E.J. Dreyer, P.A., Hedetniemi, S.M., and Hedetniemi, S.T. 2004. Roman domination in graphs, Discrete math., 278, 11 -22.

[9] Ian Stewart. 1999. Defend the Roman Empire!., Scientific American, 281(6), 136 -139.

[10] Jaya Subba Reddy. C., Reddappa, M., and Maheswari. B. 2019. Roman domination in a certain type of interval graph, International Journal of Research and analytical Reviews, 6(1), 665-672.

[11] Ore, O. 1962. Theory of Graphs, Amer, Math.Soc. Collaq.Publ.38, Providence.

[12] ReValle, C.S., and Rosing K,E. 2000. Defenden imperiumromanum: a classical problem in military.

[13] SivaParvathi, M and Maheswari, B. 2016. Minimal Signed Roman dominating functions of a corona product Graph of a path with star - International Journal of Mathematics and computer Applications Research, 6(2), $67-72$. 